\title{
Cisinta
}

\section{Analysis of Data Mining Associations on Drug Sales at Pharmacies with APRIORI Techniques}

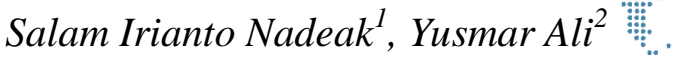 \\ ${ }^{1,2}$ Politeknik Negeri Media Kreatif, Indonesia \\ ${ }^{1}$ salamirianto77@gmail.com, ${ }^{2} y$ usalim.msi@gmail.com
}

\begin{abstract}
The purpose of the research is to utilize artificial intelligence techniques in analyzing drug sales. Sources of data used are observations and interviews with shop owners. The method used as a solution is the Association method with the Apriori technique. By using the RapidMiner software, the test results are obtained using a minimum support of 25\% and a minimum of $60 \%$ confidence as many as 5 rules with a predetermined itemset. The results of the study are expected to provide information and make it easier for related parties to find combinations of selling items. The results of this analysis can be used by pharmacies for marketing strategies and product promotion.
\end{abstract}

Keywords: Data Mining, Drug Sales, Association, Apriori Algorithm

\section{Introduction}

Sales transaction data shall be available to a pharmacy or shops selling their goods. Due to the large number of transactions occurring daily, daily transaction information will increase data. A pharmacy must have important information to boost sales by processing transaction data, which have collected useful information, in order to increase sales. This helpful data can be used for a corporate policy, namely by using the information in a corporate strategy. To develop your company, a pharmacy certainly needs a business strategy. Data mining [1], [2] is an activity that seeks out interesting patterns in large amounts of data and stores them in a data warehouse or other form of information storage. Data mining patterns must be simple to understand and practical [3]. The Apriori algorithm [4-6] is a frequent itemset search algorithm that employs association rule technology. One of the many pharmacies in the area is "United." This pharmacy is on Jalan Sangnawaluh Rasaan, not far from residential areas. "United" pharmacies always strive to provide high-quality services and products, but they still fall short of competing with other pharmacies in the area. As a result, "ABC" pharmacies must be able to comprehend consumer needs. One method is to keep various drugs available in the pharmacy warehouse. Data mining can be used for analytical technology, namely analyzing consumer needs, to determine which drugs are purchased by consumers. The Apriori algorithm can help shape the possible combinations of candidate products and then test whether those combinations meet the user's minimum support and trust parameters. Every day, there are more and more buying and selling activities for services to consumers at the "United" Pharmacy, resulting in a larger data pile. Although the "ABC" pharmacy's service activities and transactions have not encountered any issues thus far, the large amount of data collected at one time can become an impediment to improving services, making it difficult for pharmacies to analyze the types of products that are most in demand and least desired by consumers. 


\section{Cisinta}

\section{Research Methodology}

\subsection{Data Mining}

Data mining is an iterative and interactive process for discojering new patterns or models in large databases that are completely effective, useful, and easy to understand. Data mining patterns must be simple to understand, novel, and useful. Data mining is the process of discovering interesting patterns or information in selected data by employing specific techniques or methods. In data mining, the relationship sought can be one between two or more in one dimension [7].

\subsection{Association}

The Association method is to display the confidence or relationship between items. The Association method includes two stages, namely finding the most frequent combination of an itemset and defining the conditions and results (for the conditional association method). In determining an association method, there is an interestness measure obtained from the results of data processing with certain calculation data [8].

The formation of association rules consists of two steps:

a) Mode analysis at high frequencies

At this point, the database is searched for a combination of items that meet the minimum support value requirements. Support for the rule "X $=>Y$ " is the likelihood of an attribute or set of attributes $\mathrm{X}$ and $\mathrm{Y}$ appearing in a transaction at the same time.

Support $(X=>Y)=P(X \cap Y)$

$\mathrm{X} \Rightarrow \mathrm{Y}=$ items that appear in the same order $\mathrm{P}(\mathrm{XY})=$ probability of $\mathrm{X}$ and $\mathrm{Y}$ transactions divided by total number of transactions.

b) Formation of the association's rules

After locating all high-frequency patterns, compute the confidence of the if $\mathrm{X}$ then $\mathrm{Y}$ rules in order to identify association rules that meet the minimum confidence requirements..

Confidence $(X=>Y)=P(Y \mid X)$

$\mathrm{X}=>\mathrm{Y}=$ items that appear in the same order $\mathrm{P}(\mathrm{Y} \mid \mathrm{X})=$ the probability of the number of transactions that contain $\mathrm{X}$ and $\mathrm{Y}$ divided by the number of transactions that contain $\mathrm{X}$.

\subsection{Apriori Algorithm}

The Apriori algorithm was proposed in 1994 by Agrawal and Srikant to determine frequent itemsets for boolean association rules. Apriori algorithm makes use of prior knowledge about itemsets with frequent occurrences, also known as itemset frequency frekuensi [9].

\section{Result and Discussion}

The information used in this study is from drug sales in "ABC" pharmacies. In this study, 8 data samples were collected, and the collected data will be calculated by looking for support and confidence values.

Table 1. Data used

\begin{tabular}{|c|c|c|c|c|c|c|c|c|}
\hline \multirow{2}{*}{ No } & \multirow{2}{*}{ Itemset } & \multicolumn{9}{|c|}{ Day } \\
\cline { 3 - 10 } & & Monday & Tuesday & Wednesday & Thursday & Friday & Saturday & Sunday \\
\hline $\mathbf{1}$ & Antangin Jrg Cair & 60 & 56 & 55 & 50 & 60 & 15 & 22 \\
\hline $\mathbf{2}$ & Ambeven Kapsul & 55 & 10 & 52 & 56 & 55 & 15 & 21 \\
\hline
\end{tabular}




\begin{tabular}{|c|c|c|c|c|c|c|c|c|}
\hline \multirow[t]{2}{*}{ No } & \multirow[t]{2}{*}{ Itemset } & \multicolumn{4}{|r|}{ Day } & \multicolumn{2}{|l|}{ 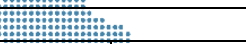 } & 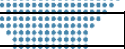 \\
\hline & & Monday & Tuesday & Wednesday & Thursday & Friday & Saturday & Sumday \\
\hline 3 & Insto Tetes Mata & 50 & 55 & 53 & 40 & 49: & 30 & 雚50 \\
\hline 4 & Albothyil $5 \mathrm{ml}$ & 40 & 40 & 45 & 49 & 50 & 10 & $\$ 5$ \\
\hline 5 & Alvita Tablet & 45 & 15 & 30 & 30 & 40 & 45 & 49 \\
\hline 6 & Andalan & 25 & 26 & 10 & 15 & $10^{\circ}$ & 25 & 20 \\
\hline 7 & Andalan Laktasi & 15 & 16 & 19 & 25 & 27 & 14 & 11 \\
\hline 8 & Alpara Tablet & 20 & 10 & 15 & 11 & 25 & 20 & 10 \\
\hline
\end{tabular}

The manual calculation of the Apriori Algorithm is as follows:

a) Transaction Patterns in Drug Sales at "ABC" Pharmacies

Based on drug sales data from "United" Pharmacies. The transaction patterns are obtained by analyzing the eight types of drugs that occur the most frequently on a daily basis, as shown in table 2 below.

Table 2. Drug Sales Transaction Pattern

\begin{tabular}{|c|l|}
\hline Day & \multicolumn{1}{|c|}{ Itemset } \\
\hline Monday & Antangin Jrg Cair, Ambeven Kapsul, Insto Tetes Mata \\
\hline Tuesday & Antangin Jrg Cair, Insto Tetes Mata, Albothyl $5 \mathrm{ml}$ \\
\hline Wednesday & Antangi Jrg Cair, Insto Tetes Mata, Ambeven Kapsul \\
\hline Thursday & Ambeven Kapsul, Antangin Jrg Cair, Albothyl $5 \mathrm{ml}$ \\
\hline Friday & Antangin Jeg Cair, Ambeven Kapsul, Albothyl $5 \mathrm{ml}$ \\
\hline Saturday & Alvita Tablet, Insto Tetes Mata, Andalan \\
\hline Sunday & Insto Tetes Mata, Alvita Tablet, Antangin Jrg Cair \\
\hline
\end{tabular}

b) Tabular Format Creation

Tabular format of daily transaction data, when formed, will look like table 3 below:

Table 3. Tabular Format of Transaction Data

\begin{tabular}{|l|c|c|c|c|c|c|c|}
\hline \multirow{2}{*}{ Itemset } & \multicolumn{7}{|c|}{ Day } \\
\cline { 2 - 9 } & Monday & Tuesday & Wednesday & Thursday & Friday & Saturday & Sunday \\
\hline Antangin Jrg Cair & 1 & 1 & 1 & 1 & 1 & 0 & 1 \\
\hline Ambeven Kabsul & 1 & 0 & 1 & 1 & 1 & 0 & 0 \\
\hline Aito Tetes Mata & 1 & 1 & 1 & 0 & 0 & 1 & 1 \\
\hline Albothyil 5 ml & 0 & 1 & 0 & 1 & 0 & 0 & 0 \\
\hline Alvita Tablet & 0 & 0 & 0 & 0 & 0 & 1 & 1 \\
\hline Andalam & 0 & 0 & 0 & 0 & 0 & 1 & 0 \\
\hline
\end{tabular}

c) High Frequency Pattern Analysis

1. Formation of 1 Itemset

The process of forming $\mathrm{C} 1$ or called 1 itemset with a minimum amount of support = $15 \%$. With the following formula:

$$
\text { Support }(\mathrm{A})=\frac{\sum \text { Transaksi yang mengandung A }}{\Sigma \text { Transaksi }} * 100 \%
$$

The following is the calculation of the formation of 1 itemset:

Support Antangin Jrg Cair $=\frac{\sum \text { Transaksi Antangin Jrg Cair }}{\Sigma 7}=\frac{6}{7} * 100 \%=85,71 \%$

Support Ambeven Kapsul $=\frac{\Sigma \text { Transaksi Ambeven Kapsul }}{\Sigma 7}=\frac{4}{7} * 100 \%=57,14 \%$

Support Insto Tetes Mata $=\frac{\Sigma \text { Transaksi Insto Tetes Mata }}{\Sigma 7}=\frac{5}{7} * 100 \%=71,42 \%$

Support Albothyl $5 \mathrm{ml}=\frac{\Sigma \text { Transaksi Albothyl Cair }}{\Sigma 7}=\frac{2}{7} * 100 \%=28,57 \%$ 
Support Alvita Tablet $=\frac{\Sigma \text { Transaksi Alvita Tablet }}{\Sigma 7}=\frac{2}{7} * 100 \%=20 \% 57 \%$

Support Andalan $=\frac{\Sigma \text { Transaksi Andalan }}{\Sigma 7}=\frac{1}{7} * 100 \%=14,28 \%$

Based on the description above, it can be made table 4:

Table 4. Support of each Itemset

\begin{tabular}{|l|c|c|}
\hline \multicolumn{1}{|c|}{ Itemset } & amount & Support \\
\hline Antangin Jrg Cair / A & 6 & $85,67 \%$ \\
\hline Ambeven Kabsul / B & 4 & $57,11 \%$ \\
\hline Aito Tetes Mata / C & 5 & $71,42 \%$ \\
\hline Albothyil 5 ml / D & 2 & $28,57 \%$ \\
\hline Alvita Tablet / E & 2 & $28,57 \%$ \\
\hline Andalan / F & 1 & $14,28 \%$ \\
\hline
\end{tabular}

2. Combination of 2 Itemset

The process of forming $\mathrm{C} 2$ or called 2 itemset with a minimum amount of support $=$ $20 \%$. With the following formula:

$\operatorname{Support}(\mathrm{A}, \mathrm{B})=\frac{\Sigma \text { Transaksi yang mengandung } A, B}{\Sigma \text { Transaksi }} * 100 \%$

The following is the calculation of the formation of 2 itemsets:

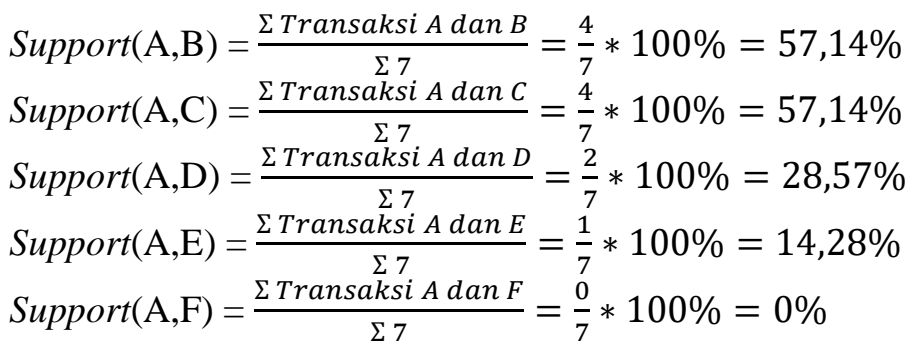

The minimum Support specified is $20 \%$, so the combination of 2 itemsets that do not meet the minimum Support will be removed, as shown in Table 5 below:

Table 5. Minimum Support 2 itemset 20\%

\begin{tabular}{|l|c|c|}
\hline \multicolumn{1}{|c|}{ Itemset } & amount & Support \\
\hline Antangin Jrg Cair, Ambeven Kabsul & 4 & $57,14 \%$ \\
\hline Antangin Jrg Cair, Aito Tetes Mata & 4 & $57,14 \%$ \\
\hline Antangin Jrg Cair, Albothyil 5 ml & 2 & $28,57 \%$ \\
\hline Ambeven Kabsul. Aito Tetes Mata & 2 & $28,57 \%$ \\
\hline Aito Tetes Mata, Alvita Tablet & 2 & $28,57 \%$ \\
\hline
\end{tabular}

3. Combination of 3 Itemset

The process of forming $\mathrm{C} 3$ or called 3 itemset with a minimum amount of $20 \%$. With the following formula:

$\operatorname{Support}(\mathrm{A}, \mathrm{B}$ dan $\mathrm{C})=\frac{\sum \text { Transaksi yang mengandung } A, B \text { dan } C}{\Sigma \text { Transaksi }} * 100 \%$

The following is the calculation of the formation of 3 itemset:

Support (A,B dan C) $=\frac{\Sigma \text { Transaksi } A, B \text { dan } C}{\Sigma 7}=\frac{2}{7} * 100 \%=28,57 \%$

$\operatorname{Support}(\mathrm{A}, \mathrm{B}$ dan $\mathrm{F})=\frac{\Sigma \text { Transaksi A,B dan } F}{\Sigma 7}=\frac{0}{7} * 100 \%=0 \%$ 
Support $(\mathrm{A}, \mathrm{C}$ dan $\mathrm{D})=\frac{\sum \text { Transaksi } A, C \text { dan } D}{\Sigma 7}=\frac{1}{7} * 100 \%=14,28 \%$

Support $(\mathrm{A}, \mathrm{C}$ dan $\mathrm{E})=\frac{\sum \text { Transaksi A,C dan } E}{\Sigma 7}=\frac{0}{7} * 100 \%=0 \%$

$\operatorname{Support}(\mathrm{B}, \mathrm{D}$ dan $\mathrm{F})=\frac{\Sigma \text { Transaksi } B, D \text { dan } F}{\Sigma 7}=\frac{0}{7} * 100 \%=0 \%$

Based on the description above, it can be made table 6 :

Table 6. Combination of 3 itemset

\begin{tabular}{|l|c|c|}
\hline \multicolumn{1}{|c|}{ Itemset } & Jumlah & Support \\
\hline Antangin Jrg Cair, Ambeven Kapsul, Insto Tetes Mata & 2 & $28,57 \%$ \\
\hline Antangin Jrg Cair, Insito Tetes Mata, Albothyil 5 ml & 1 & $14,28 \%$ \\
\hline Antangin Jrg Cair, Ambeven Kapsul, Andalan & 1 & $14,28 \%$ \\
\hline Aito Tetes Mata, Alvita Tablet, Andalan & 1 & $14,28 \%$ \\
\hline Antangin Jrg Cair, Aito Tetes Mata, Alvita Tablet & 1 & $14,28 \%$ \\
\hline
\end{tabular}

The minimum support specified is $25 \%$, then 3 combinations that meet the requirements will form an association rule.

d) Rules for Association Formation

After identifying all high-frequency patterns, the association rules that meet the minimum Confidence requirements are found by calculating the Confidence of the associative rules $\mathrm{AB}$ with a minimum confidence value of $60 \%$. The following formula yields the $\mathrm{AB}$ rule's confidence value:

Confidance $=\mathrm{P}(\mathrm{A} / \mathrm{B})=\frac{\sum \text { Transaksi yang mengandung } A}{\Sigma \text { Transaksi } A} * 100 \%$

The following is a Confidence calculation:

$$
\begin{aligned}
& \text { Confidance }=\mathrm{P}(\mathrm{A} / \mathrm{B})=\frac{\Sigma \text { Transaksi } A \text { dan } B}{\Sigma 6}=\frac{4}{6} * 100 \%=66,66 \% \\
& \text { Confidance }=\mathrm{P}(\mathrm{A} / \mathrm{C})=\frac{\Sigma \text { Transaksi } \mathrm{A} \text { dan } C}{\Sigma 6}=\frac{4}{6} * 100 \%=66,66 \% \\
& \text { Confidance }=\mathrm{P}(\mathrm{A} / \mathrm{D})=\frac{\Sigma \text { Transaksi } \mathrm{A} \text { dan } D}{\Sigma 6}=\frac{2}{6} * 100 \%=33,33 \% \\
& \text { Confidance }=\mathrm{P}(\mathrm{A} / \mathrm{E})=\frac{\sum \text { Transaksi } \mathrm{A} \text { dan } E}{\sum 6}=\frac{1}{6} * 100 \%=16,66 \% \\
& \text { Confidance }=\mathrm{P}(\mathrm{A} / \mathrm{F})=\frac{\Sigma \text { Transaksi } \mathrm{A} \text { dan } F}{\Sigma 6}=\frac{0}{6} * 100 \%=0 \%
\end{aligned}
$$

From the combination of 2 itemsets that have been determined, it can be seen the magnitude of the support value and the confidence value of the candidate association as shown in the following table:

Table 7. Candidate Association Rules

\begin{tabular}{|l|c|c|}
\hline \multicolumn{1}{|c|}{ Rules } & \multicolumn{2}{c|}{ Confidence } \\
\hline If you buyAntangin Jrg Cair Then Will Buy Ambeven Kabsul & $4 / 6 \%$ & $66,66 \%$ \\
\hline If you buyAntangin Jrg Cair Then Will Buy Aito Tetes mata & $4 / 6 \%$ & $66,66 \%$ \\
\hline If you buyAntangin Jrg Cair Then Will Buy Albothyil 5 ml & $2 / 6 \%$ & $33,33 \%$ \\
\hline If you buyAntangin Jrg Cair Then Will Buy Alvita Tablet & $1 / 6 \%$ & $16,66 \%$ \\
\hline If you buyAntangin Jrg Cair Then Will Buy Andalan & $0 / 7 \%$ & $0,00 \%$ \\
\hline If you buyAmbeven Kabsul Then Will Buy Aito Tetes mata & $2 / 4 \%$ & $50 \%$ \\
\hline If you buyAmbeven Kabsul Then Will Buy Albothyil 5 ml & $1 / 4 \%$ & $25 \%$ \\
\hline If you buyAmbeven Kabsul Then Will Buy Alvita Tablet & $0 / 4$ & 0 \\
\hline If you buyAmbeven Kabsul Then Will Buy Andalan & $0 / 4 \%$ & 0 \\
\hline If you buyAito Tetes Mata Then Will Buy Albothyil 5 ml & $1 / 5 \%$ & $20 \%$ \\
\hline
\end{tabular}




\begin{tabular}{|c|c|c|c|}
\hline Rules & 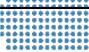 & \multicolumn{2}{|c|}{ Confidënence } \\
\hline If you buyAito Tetes Mata Then Will Buy Alvita Tablet & & $2 / 5 \%$ & 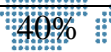 \\
\hline If you buyAito Tetes Mata Then Will Buy Andalan & ") & $1 / 5 \%$ & $20 \%$ \\
\hline If you buyAlbothyil $5 \mathrm{ml}$ Then Will Buy Alvita Tablet & ;... & $0 / 2 \%$ & 0 \\
\hline If you buyAlbothyil $5 \mathrm{ml}$ Then Will Buy Andalan & & $0 / 2 \%$ & $0 \%$ \\
\hline If you buyAlvita Tablet The & & $1 / 1 \%$ & $100 \%$ \\
\hline
\end{tabular}

e) Final Association Rules

Based on the candidate association rules in table 7, those who meet a minimum of $25 \%$ support and a minimum of $60 \%$ confidence can be seen in the table below:

Table 8. Final Association Rules

\begin{tabular}{|l|c|c|}
\hline \multicolumn{1}{|c|}{ Rules } & Support & Confidence \\
\hline If you buyAntangin Jrg Cair Then Will Buy Ambeven Kabsul & $57.14 \%$ & $66,66 \%$ \\
\hline If you buyAntangin Jrg Cair Then Will Buy Aito Tetes mata & $57,14 \%$ & $66,66 \%$ \\
\hline If you buyAntangin Jrg Cair Then Will Buy Albothyil 5 ml & $28,57 \%$ & $33,33 \%$ \\
\hline If you buyAmbeven Kabsul Then Will Buy Aito Tetes mata & $28,57 \%$ & $50,00 \%$ \\
\hline If you buyAito Tetes Mata Then Will Buy Alvita Tablet & $28,57 \%$ & $40,00 \%$ \\
\hline
\end{tabular}

\section{Conclusion}

From the test results using a minimum support of $25 \%$ and a minimum confidence of $60 \%$, it produces a possible rule.

a) If you buyAntangin Jrg Cair, Then Will Buy Ambeyen Kapsul generate value support $57,14 \%$ and confidence $66,66 \%$.

b) If you buyAntangin Jrg Cair, Then Will Buy Insto Tetes Mata generate value support $57,14 \%$ and confidence $66,66 \%$.

c) If you buyAntangin Jrg Cair, Then Will Buy Albothyl $5 \mathrm{ml}$ generate value support 28,57\% and confidence 33,33\%.

d) If you buyAmbeyen Kapsul, Then Will Buy Insto Tetes Mata generate value support $28,57 \%$ and confidence $50,00 \%$.

e) If you buyInsto Tetes Mata, Then Will Buy Alvita Tablet generate value support $28,57 \%$ and confidence $40,00 \%$.

\section{References}

[1] A. P. Windarto, U. Indriani, M. R. Raharjo, and L. S. Dewi, "Bagian 1: Kombinasi Metode Klastering dan Klasifikasi (Kasus Pandemi Covid-19 di Indonesia)," Jurnal Media Informatika Budidarma, vol. 4, no. 3, p. 855, 2020, doi: 10.30865/mib.v4i3.2312.

[2] A. P. Windarto, J. Na, and A. Wanto, "Bagian 2: Model Arsitektur Neural Network dengan Kombinasi K- Medoids dan Backpropagation pada kasus Pandemi COVID-19 di Indonesia," vol. 4, pp. 1175-1180, 2020, doi: 10.30865/mib.v4i4.2505.

[3] S. Kasus, P. Pt, G. Gunadi, and D. I. Sensuse, "Penerapan Metode Data Mining Market Basket Analysis Terhadap Data Penjualan Produk Buku Dengan Menggunakan Algoritma Apriori Dan Frequent Pattern Growth (Fp-Growth):," vol. 4, no. 1, 2012.

[4] R. D. Jayapana and Y. Rahayu, "Analisis pola pembelian konsumen dengan algoritma apriori pada apotek rahayu jepara," $U G$ Jurnal, vol. Vol. 8, pp. 1-6, 2015 . 
[5] N. Agustiani, D. Suhendro, K. Apriori, and A. Pembathasain: "Penerápanu Data

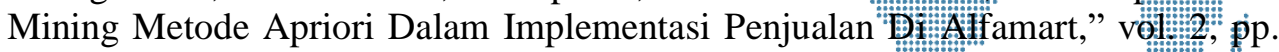
300-304, 2020.

[6] H. Jantan and A. Z. M. Jamil, "Association rule mining based crime analysis using apriori algorithm," International Journal of Advanced Trends' in Computer Science and Engineering, vol. 8, no. 1.5 Special Issue, pp. 18-24, 2019, doi: 10.30534/ijatcse/2019/0581.52019.

[7] P. Obat and M. Algoritma, "Data mining," vol. 2, no. 2, pp. 1-7, 2013.

[8] Putri Septiani Azura, M Safii, and Fitri Rizki, "Analisa Penjualan Produk Oli Dengan Metode Data Mining," vol. 2, no. 1, pp. 8-15.

[9] Dewi Listriani, Anif Hanifa Setyaningrum, and Fenty Eka M.A, "Penerapan Metode Asosiasi Menggunakan Algoritma Apriori Pada Aplikasi Analisa Pola Belanja Konsumen ( Studi Kasus Toko Buku Gramedia Bintaro )," vol. 9, no. 2, pp. 120-127, 2016. 